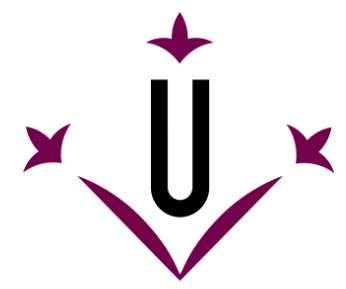

Universitat de Lleida

Document downloaded from:

http://hdl.handle.net/10459.1/69057

The final publication is available at:

https://doi.org/10.1080/1369183X.2017.1341709

Copyright

(c) Taylor and Francis, 2018 


\section{Reciprocity for new migrant integration: resource conservation, investment and exchange}

\section{Abstract}

In this paper we bring a new perspective to the understanding of migrant integration. We focus on how new migrants use reciprocity to make and sustain connections. In turn, we identify integration resources accessed through those connections and associated acts of reciprocal exchange. Using qualitative data collected in retrospective interviews from a maximum variation sample of new migrants arriving in the UK up to two years before interview we identify five interconnected sub-types of reciprocity and explore how these are used to replace or substitute resources lost through the act of migration. We argue that, contrary to Hobfoll's (2011) ideas about conservation of resources in crisis, migrants use resource exchange strategies to develop social networks which may form important buffers against migratory stress and aid access to functional, psychological and affective resources that can further integration. The paper concludes by highlighting the significance of reciprocity in moving the theorisation of integration in new a new direction.

Key words: Migrants, integration, reciprocity, resources

\section{Introduction}

The movement of peoples from the global south to the industrialised north has contributed to the superdiversification of Europe and fuelled moral panic and widely articulated fears that migrants place a burden on societies that exceeds their economic contribution. Anxiety about migrants allegedly co-opting resources such as social benefits, housing, healthcare and jobs while refusing to mix with local people or adapt 
to the frequently homogenised and prescriptive values and cultures of nation states abound (Kremer 2016). Even countries such as the Netherlands, Sweden and the UK, which for decades supported a multicultural approach to acculturation, have experienced a backlash (Vertovec \& Wessendorf 2010). This trend was most recently illustrated by the outcome of the UK's Brexit referendum in which the majority of the UK electorate that voted, opted to leave the European Union in a decision widely described by politicians as a decision to curtail immigration from Europe. Increased anti-migration rhetoric from politicians and the media have been coupled with calls for migrants to integrate into their host societies (Grzymala-Kazlowska and Phillimore, 2017). Closer investigation of these demands indicates that calls for integration are in fact demands for assimilation (Bloemraad et al., 2008; Brubaker, 2001; Triadafilopoulos, 2011). These include adaptation to national values and behaviours and for migrants to contribute to civil society, rather than, as perceived, utilising scarce national resources.

There has been little acknowledgement in this discourse that migrants, in the process of migration, often make enormous sacrifices leaving behind resources such as home, friends, family, status and agency, that most sedentary people take for granted. However there is considerable consensus that varied social networks are critical to new migrants helping them overcome "bereavement" and contributing to integration processes (see Phillimore, 2013) partly because networks frequently enable access to social capital: resources that would otherwise be unavailable to them (Foley and Edwards, 1999). While policymakers have expressed concerns about the nature of migrant networks, with those formed with co-ethnics seen as problematic because they are believed to promote self-segregation, there is clear evidence that networks matter (Anthias and Cederberg, 2009; Barwick, 2015). Possessing extensive and varied 
networks has been found to facilitate integration in a wide range of domains including employment, housing, access to health and education. Resource exchange has long been recognised as one of the main mechanisms utilised to build new networks following Mauss's seminal book The Gift which arguably forms the foundation of social theories of reciprocity. Reciprocity, defined in the Oxford English Dictionary (2016) as "the practice of exchanging things with others for mutual benefit” is argued by Mauss and subsequently Simmel to be the basis of society and of key importance in ensuring social cohesion. Little is known about the mechanisms that connect the forming of networks with reciprocity, and access to new resources.

In this paper we bring an original new lens to the understanding of migrant integration through drawing on interviews conducted with new migrants who had been living in the UK for less than two years. We focus on how these migrants used reciprocity to make and sustain connections. We identify the integration resources they accessed through both their social connections and the act of exchange in itself. We argue that although newcomers might be considered resource poor and could be expected to, as Hobfoll (2011) suggests, conserve their resources, they use different resource exchange strategies to develop social networks which may form important buffers against migratory stress and aid access to resources that can further integration. The paper begins by exploring the literature around migration and loss of resources using Hobfoll's Conservation of Resources (COR) theory to provide a basis for understanding the nature of resources before outlining five interconnecting forms of reciprocity and how these might relate to migrant integration. We then describe the methodology used to collect data with migrants and examine approaches to reciprocity adopted and resources exchanged and gained. The paper concludes with a discussion about the significance of reciprocity in extending thinking about integration. 


\section{Migration, resource loss and stress}

While many of the concerns expressed about the increase in the numbers of migrants arriving to Europe have focused upon pressure they allegedly place on scarce resources (Migration Advisory Committee, 2012) there has been less emphasis on the loss of resources experienced by migrants (or the resources that they bring with them). This experience is recognised by social-psychologists to be so intense that it has been termed migratory grief (Casado et al., 2010). While individuals' experiences of migration are highly varied and some migrants (for example highly skilled migrants) benefit more than others, the process of separation from country of origin often involves the loss of families, friends, and language (Henry et al., 2005) and symbolic resources such as landscape, music, weather, and media (Markovitzky and Mosek, 2005). Migration may represent a new beginning or potential to gain new opportunities which may mitigate feelings of loss, however, the sense of loss can also affect psychological adjustment to new environments and result in post-migratory stress. Some kind of continuing bond to "home" and those who reside there provides resources which help with adjustment to a new life, problem solving or solace (Henry et al., 2009).

Much has been written about migrant adaptation to new circumstances, frequently termed in social-psychology as acculturation. Berry (2009) views acculturation as a psychological process with integration, which he defines as a twoway adaptation of the so-called dominant (i.e. host) community and non-dominant (migrants), seen as the most positive acculturative pathway. Such mutual adjustment ideally supports non-dominants to retain aspects of their own culture thereby helping to reduce post-migratory bereavement and gradually adapt to the dominant culture, while institutions and dominant populations adjust to meet new needs and accept new cultures. Berry (2005) argues integration is the least stressful acculturative process for 
migrants as it enables them to use the resources offered by continuing bonds to establish some stability during adaptation, and thus reduce stress. Bhatia and Ram (2009) however argue there is no single process of adaptation and no endpoint. They show how Pakistani minorities born in the US and believing themselves fully integrated were made to feel "other" following racist harassment in the aftermath of the 9/11 attacks. This experience, they suggest, indicates that acculturation is ongoing, contested and negotiated, impermanent and intersubjective and can reverse as well as proceed. They portray individuals as agents engaged in a process of integration and subject to influences, experiences and resources that may impede or facilitate their journey. In order to engage in integration processes individuals must access wide ranging resources - the presence or absence of which can shape opportunities and trajectories (see Grzymala-Kazlowska 2017). For the purposes of this paper we define integration as the processes of adjustment undertaken by migrants in order to adjust to life in a new country ${ }^{1}$.

Hobfoll (2011:339) defines resources as “objects, personal characteristics or energies that are valued in their own right, or that are valued because they act as conduits to the achievement or protection of valued resources”. Examples of resources include feelings, intimacy, agency, money, independence and control and assets, stability, help and belonging (see Table 1). Loss of resources is critical in the stress process and of clear relevance to migrants who as we establish above abandon wide ranging resources in order to migrate. The extent to which individuals choose to

\footnotetext{
${ }^{1}$ We focus on new migrants in this paper and thus outline a definition of integration for migrants however we continue to argue as we have in earlier work that integration concerns the processes of adjustment undertaken by migrant and longer term residents.
} 
migrate and thus make a decision to part with resources varies, with forced migrants often unable to take anything with them, and others having various degrees of agency, depending upon structural and legal status factors. The Conservation of Resources (COR) theory has been employed widely to understand the ways in which individuals utilise resources to recover from crisis (Benight et al., 1999; Chen and Wen, 2010; Frydenberg, 2014; Halbesleben et al., 2014; Hobfoll, 2011). Hobfoll provides extensive evidence that "personal, social and economic resources can be invested to aid the process of stress resistance” (349). COR has been used to examine how resources have been employed in relation to recovery from environmental disaster, sexual assault, workplace stress, and addiction amongst other areas but as yet the issue of resources and how they are utilised following migration has received no attention. We argue that it is possible to conceive of integration processes as attempts to replace or substitute resources lost through migration and utilised in order to get on socially and economically while reducing acculturative stresses. Evidence suggests that following resource loss individuals utilise a range of recovery strategies with the primary intention being to re-establish self-esteem and reduce stress through resource replacement (Benight et al., 1999; Chen and Wen, 2010; Frydenberg, 2014; Halbesleben et al., 2014; Hobfoll, 2011 see Grzymala-Kazlowska 2017). Alternatively, individuals attempt resource substitution by replacing lost resources with others of equivalent value (Hobfoll 2011).

\section{Table 1 about here}

Migration scholars have given much consideration to categorising indicators of integration with Ager and Strang (2008) identifying four domains. These include functional resources such as housing, education, health and employment which they describe as means to integration and markers of being integrated; social connections in 
the form of bridges, bonds and links; facilitators such as language, cultural knowledge, safety and stability and foundations in terms of rights and access to citizenship. While there is empirical evidence of the importance of these indicators and some connect well with Hobfoll's resource descriptors the role of wider resources such as intimacy, selfesteem and agency are over-looked. Yet these are clearly important in helping migrants to cope with acculturative stress as Abdulahad et al., (2012) show looking at Iraqi Christians in Ontario where they found stress was associated with loss of esteem and identification with a familiar country. Building on Bhatia and Rams' (2009) contention about the importance of agency and upon Hobfoll's argument about the role of resources in avoidance of stress, we contend that it is important to understand how migrants use resources for integration purposes. The level of resources possessed on arrival is important, as Hobfoll argues those with more resources are less vulnerable to future resource losses. His COR theory posits that those with least resources are aware of their vulnerability to further resource loss and will conserve their resources through adopting a defensive position. Thus migrants could be expected to consolidate resources in some integration domains until they are somewhat established in others.

\section{Conservation, reciprocity or exploitation?}

Those who experience a decline in their personal resilience after a crisis have increased propensity to stress and are more vulnerable to future stressors with resource exchange, which Mauss labels reciprocity, emerging as particularly important in buffering against stress. It is important to consider further the nature of reciprocity and how it might relate to integration. Mauss’s (1954) seminal study highlighted the importance of resource exchange in the form of gift-giving, in developing and sustaining social connections and indeed portrays the giving of such resources as almost a strategic approach to acquiring other resources. Resource exchange bestows authority 
and prestige while refusal to exchange can be viewed as a rejection of a relationship and the social norms that have conditioned it. Simmel suggests that reciprocity through exchange is the basis of network formation while Papilloud (2004) contends that a combination of sacrifice, reciprocity and duration shape the possibility of human relations. Exchange involves an initial sacrifice: parting with something of value, but also the risk that the gift might be spurned or not-reciprocated (Möllering, 2001). Frank \& Yasumoto (1998) show the importance of duration in that repeated acts of resource exchange enable "norms and behaviour to co-evolve" thus offering potential for the development of new cultural understandings and the two-way adaptation of norms and behaviours that Berry argues is so important for integration.

Reciprocity must occur over sufficient duration to cement a social relationship. Friendship, as an intimate form of social relationship, can be considered a key resource in integration (Ager and Strang, 2008). Friendships have value for migrants as a source of intimacy and solace from migratory stress but also friendship networks can in themselves provide access to resources, often described as social capital (Foley and Edwards, 1999). Social networks have been associated with enhanced access to functional integration resources such as housing, health and employment (Phillimore, 2013; Ryan 2017). So ability to exchange resources with others is particularly important for new migrants to help develop social networks and capital (Molm, 2010). But in order to participate in reciprocity some level of resource must be available to exchange. Many newly arrived migrants, experiencing the crisis that is the resource loss associated with migration, often arrive with low levels of resources so may be unable to exchange thereby rendering them more susceptible to acculturative stress and reducing their potential to form social networks. 
Komter (2007) argues that resource exchange supports the development of mutual loyalty and has potential to connect those who are hostile to each other. With migrants arriving into new and increasingly unreceptive contexts, and the importance of social networks for developing a sense of psychological well-being (Phillimore, 2013) it is clear that resource exchange has potential to support integration and perhaps even reduce enmity. Through offering resources migrants might enjoy receiving gratitude and the associated moral obligations that form the basis of trust thereby securing new social relationships through building solidarity with those who undertake supportive exchanges as relationships intensify over time (Komter, 2007). Bilecen’s (2014) work shows that the strength of emotional connections determine willingness to provide or ask for assistance - the stronger the ties the more willing to exchange. The desire for what we term herein, norm-based reciprocity, founded upon repeated and more or less balanced resource exchange between known others is a critical psychological concern that impacts upon stressors such as self-esteem and self-efficacy. As such engaging in norm-based reciprocity may have potential to reduce acculturative stress and enable access to resources that can support integration processes. However not all reciprocity is norm-based or balanced.

It is possible to distinguish between different sub-types of reciprocity, with each potentially shaping integration processes in different ways. Godbout's (2005) notion of informal reciprocity (in Bernard et al., 2007) describes exchange of resources given freely by individuals to other individuals, particularly to strangers as we show below. It is not known if repayment will occur, or even if the same person will be repaid. This lack of direct repayment could imply a relationship was exploitative but Bernard et al. (2007) argue relationships emerging from the act of exchange are at least as important as the exchange itself. Informal reciprocity is based around the idea that trust at local or 
community level will lead to a reciprocal action at some point. Systems of informal reciprocity are open-ended and thus in a perpetual state of imbalance. Bernard et al (2007:1847) argue informal reciprocity offers those without much power an opportunity to enjoy some agency, affect local social relations and 'fulfils the need for sociability, information and social support' especially amongst strangers. Thus informal reciprocity offers considerable potential for migrant integration as a means to gain support when short of resources, to develop social connections, to attain some aspects of identity through re-establishing agency when offering resources and thus operate as a mechanism for the development of self-esteem potentially substituting pre-migration psychological resources.

Nahun-Shani \& Bamberger’s (2011) study of the way social support buffers against the harmful effects of workload stress on employee well-being offer other distinctions in reciprocal relations with potential to help us understand migrant integration. They argue the extent to which reciprocity can help buffer against stress depends on the pattern of exchange relations and whether exchange is perceived as balanced, under, or over-reciprocating. They find that balanced norm-based reciprocity enhances the value of the support received by promoting a sense of identity and belonging, positive self-image and a sense of control. Where under-reciprocation occurred, that is an individual receives fewer resources than they give where some form of exchange is expected, a sense of agency is created, with clear potential for migrant integration. But 'violation of reciprocity norms may be harmful to the recipients' sense of identity and belonging', leaving individuals feeling exploited and unable to regain important resources such as belonging. Finally, over-reciprocation where individuals receive more support than they give, which might be conceived as one side of an informal reciprocated exchange, may be harmful to self-image because it creates a sense 
of dependency undermining agentic aspects while also impacting negatively on identity and belonging because exchange norms are violated when, unlike with informal reciprocity, exchange of resources is expected. It is possible that migrants without the capacity to reciprocate will lack key resources such as self-esteem and independence which may well affect levels of post-migratory stress and potential to access other integration resources.

Clearly exchange of resources is an important aspect of everyday social life and migrants will need to engage in exchange in order to build new relationships and to access replacement or substitute resources. However, there is a paradox. Hobfoll (2011) suggests that when individuals encounter a stressful situation, such as migration, they will conserve resources to avoid further stress, to build resilience for future recovery and to participate in the ongoing project of resource acquisition that is integration. With few or depleted resources migrants are unable to participate in resource exchange a situation that we term no-reciprocity. This paper uses an analytical framework based on the five interconnecting forms of reciprocity we have identified above: informal reciprocity, norm-based reciprocity, under-reciprocity, over-reciprocity and noreciprocity. The paper is innovative in that it is the first to develop the link between forming networks, reciprocity and access to new resources. Specifically, we examine the ways in which migrants engage in, or avoid, different forms of reciprocation and consider how reciprocity may contribute to migrant integration.

\section{Methods}

The data utilised in this paper comes from an European Integration Fund sponsored study, the Knowledge into Integration Governance (KING) project informing a review of the European Union's Common Basic Principles for Migrant Integration. 
The study explored the ways in which new migrants utilised networks to access resources which could further their integration. Having received full ethical approval from the University of Birmingham Ethical Review Committee, we used a maximum variation sample approach, wherein a small number of units or cases were selected that maximize the diversity relevant to the research issue. Selecting respondents who were as different from each other as possible helped to capture the superdiversity evident in many UK cities and enabled the identification of commonalities which offer potential for generalisation (Patton 2005). Having gained consent we interviewed 29 new migrants all of whom had been resident in the UK less than two years. This timeframe meant respondents were sufficiently new to be able to recall the networks they had made and used since arrival.

We took a range of approaches to locate interviewees approaching migrant support organisations in four superdiverse urban areas, a college and a local authority equality and diversity team, researchers' personal networks, word of mouth and directly approaching people in public places. Using organisations and networks means that most respondents inevitably had some kind of network however our retrospective approach worked to our advantage enabling us to understand network formation in the period before they made that connection. Clearly we were dependent on the selective memories of respondents. It's highly likely that they had experiences which they either could not, or did not want to, recall. It is likely too, that there are migrants who are completely unconnected whose perspectives we could not include in our study.

Eighteen respondents were male and eleven were female (see Appendix). Ages ranged from twenty-two to sixty-one with the majority of interviewees in their twenties. Four interviewees were claiming asylum; seven had been granted refugee status, 12 had migrated to join a spouse; two were students and four were economic migrants. They 
came from 14 different countries, 13 were living with a family member and 20 spoke English. The majority of spousal migrants had little migration experience and had moved directly from their previous place of residence to the UK. Two male spousal migrants had a large amount of migration experience before settling in the UK with their wives. Asylum seekers and family reunification migrants had a wide range of migration experiences including living in refugee camps for a decade (Somali family reunification migrant) or Syrian refugees who travelled through Turkey and Europe to claim asylum in the UK. Interviews were either undertaken in English, in the respondents' mother tongue which was spoken by one of our researchers, or with the aid of an interpreter who was identified by the community organisation. Data were coded using a systematic thematic analysis approach (Guest 2012) to identify the key issues raised by respondents (see Table 2). This involved interpretive code-and-retrieve methods wherein the data was transcribed and read by the research team who together identified codes and then undertook an interpretative thematic analysis. The quotations used in this paper were selected on the basis of their ability to illustrate those issues. All names used are pseudonyms.

\section{Reciprocity and resources}

\section{Norm-based reciprocity}

A number of respondents arrived knowing only their spouse, a single friend or relative. In the early stages of their lives in the UK they were heavily dependent on this person and spoke of feeling isolated. Most rapidly began to form friendships with other migrants, not necessarily from the same country of origin, who they were introduced to by their contact or met independently in public places. Norm-based reciprocity formed the basis for these emergent social relations with each person helping the other initially 
through exchange of information or language skills then followed by emotional and practical support. For example, Salma, a Moroccan national, came to join her Bengali husband in London knowing only him. She met her husband through the website ‘muslima.com' having previously been working in the hire car industry in Morocco and residing with her family. No family members lived in the UK. Her husband suggested she enrol for an ESOL class where she met Nada, also a spousal migrant from Morocco, who arrived shortly before Salma knowing only her husband. They rapidly developed a friendship with Salma receiving emotional support and information about how to access services from Nada and Salma reciprocating with language assistance - her English skills meant she had some resources to exchange

Yes. She has helped me a lot. If I have a problem, she has helped me. If I have a problem then I call her and she helped me. I help her for the language. If she can't understand something. First time I find life here very difficult. She talked with me, she gave me advice and support. She was here before she explained to me about the GP. She told me what they are going to ask me.

Amita's position was similar to Salma. She was highly proficient in English, had an MBA in Business and had come to join her busy husband. He introduced her via e-mail to his friend's wife, Leela who emigrated two years earlier from Pakistan, before Amita migrated. Leela advised Amita which clothes to bring with her and once she arrived they frequently exchanged resources

A: She is not my best friend but she is a friend. Because we don't have our relatives with us. So if there is any immediate problem where we need our relatives with us in such cases then she helps me with that.

I: Can you give me an example? 
A: So there I used to help her when her daughter's swimming classes finish at 4:15pm and her son finishes school at 4pm. So I was helping her - he comes to my house and he stays there. Some kind of basic help. He introduced me to her so that we became friends and now if we have anything like an emergency then she helps me and I help her.

Through shared information about job opportunities, local ways of life, and offers of emotional and practical support such as childcare they rapidly develop a close relationship within which they begin to rely on each other's help with settling into a new country.

\section{Informal reciprocity}

With just three exceptions all respondents were engaged in some degree of informal reciprocity. Providing assistance to others was seen by interviewees, as Godbout (2005) suggests, as a mechanism for investing in some kind of universal pot perhaps thinking that they might one day benefit in some way from their good deed. An example of this is the actions of Fatima, a Saudi Arabian spousal migrant of Eritrean and Egyptian heritage. After separating from her husband she developed close friendships with women in the hostel where she lived with her baby. She and her friends engaged in a balanced exchange of resources such as information and support with childcare. However, she also told of how she helped physically frail strangers

Sometime when I am going to shopping and some people can't walk and some problems in the legs. You know when you go like that one and it is very difficult and already I have some pain in my back. I am thinking for myself with my daughter I am carrying her upstairs I need some help so I am thinking if you can't help why not? Because I know when you go down and up and down and up 
and if you can't help, why not? Maybe in the future there is some problem with my mum and dad maybe and maybe some people they help her.

Offering assistance gave Fatima a sense of purpose enabling her to feel that she was valuable to others, particularly important since her family had disowned her. This kind of spontaneous informal reciprocity was commonly reported with migrants regaining self-esteem though having sufficient resources, all be they time or confidence, to make a sacrifice for complete strangers.

We commonly encountered migrants who gave time, shared information, offered their language skills and sometimes their limited financial resources to strangers. A key factor here was length of time in the UK. Those who were recently arrived had little to offer - no knowledge and some lacked language abilities - they were frequently the recipients of informal reciprocity. As they became more experienced and acquired some resources their ability to form relationships evolved. They repaid resources but crucially not with those who had originally helped them but to strangers. Thus as Godbout suggests the exchange was open ended and a matter of the relatively more established migrants exchanging with the newest.

Adil was an asylum seeker originating in Pakistan who lived with a friend in Birmingham. He was very sociable and had met many people, whom he described as friends, while shopping, at the masjid and at celebrations. Since arriving in the UK he had received hospitality from fellow Kashmiris and Muslims from South Asia and Africa. Through his extended network, a set of relationships with co-religious individuals, he was given help with orientation and legal advice, invited for dinner and to weddings, and offered work. When we met him he felt established and helped new arrivals both in person and via his mobile telephone on which completely unknown new 
migrants frequently called. In this way he sought to repay the resources he received on arrival

I: $\quad$ Do you help anyone in any way?

A: $\quad$ Yes, yes, yes. If someone comes to me and tells me for good experience I tell them where to go and all these information. I tell them my friends when they come you look after me and definitely I help them.

Fikru, a refugee from Eritrea was dispersed to Birmingham completely alone and was offered extensive resources from local people. He was startled by the levels of support in the form of advice, food and clothes he has received

I am doing because when I come this country. I seen different things. Good things. People ready to help you. When you go outside people help you. When I ask them the road......

After living in the UK for nine months he was ready to return resources and frequently offered help to people he met in the street or at college 'People when new people comes he wants to know this place, I want to show him'.

Repaying the gift was a signifier of having, as Hobfoll (2011) suggests, reacquired some resources. These more established individuals now had knowledge of how systems worked, enough time to spare, sufficient confidence to interact with strangers often from different countries of origin and enough language capability to translate for them. By repaying the resources which they benefitted from on arrival they arguably received new resources: some of the self-esteem and sense of pride that had been diminished through migration. Perhaps too they gained a sense of authority through possessing resources that strangers, as yet, did not have, as they experienced a shift of roles from relative helplessness to helpfulness. 


\section{Under-reciprocation}

We found just one individual who might be described as under-reciprocating, although arguably informal reciprocity for new arrivals inevitably begins with a period of under-reciprocity as individuals conserve resources until they are in a position to repay their part of the open-ended informal contract. Dalmar, a Somali national, arrived from a refugee camp in Kampala one year prior to interview having gained entry into the UK on a family reunion visa to join his daughter. He was completely without functional resources having no recourse to public funds, speaking no English, not permitted to work and unable to live with his daughter in her tiny shared flat. Aged 55 he struggled to cope and depended upon other Somalis, whom he made friends with at the mosque, for food and housing. He appeared to accept this under-reciprocity explaining it was part of Somali culture. Once he was more established he hoped to be in a position to help those newer than him.

And I said I don't have place to stay and he said come with me. Somalis are always like this. We like to eat together and sleep together. We talk together. We have a very good culture. It is an open door.... am the one that needs help now! No one has come after me from Somalia - I am the newest one.

In his position Dalmar lacked choice - he had few resources to conserve or exchange but this did not appear to affect his self-esteem. He showed some signs of post-migratory stress resulting from being separated from key cultural and social resources as outlined by Henry et al. (2005). However, the social bonds he developed with his friends and his acceptance of under-reciprocation may have helped him to cope with his lack of resources.

Elsewhere we noted that respondents actively sought to avoid underreciprocating considering the receipt of too many resources as a violation of the norms 
of exchange. Thus we heard from a single Syrian refugee, dispersed six months previously to Wolverhampton where he had made few close friends, about his extreme loneliness. He would not spend time with his friends or share his problems because they were married and he felt they had important resources (intimacy, independence) the conservation of which he perceived as their priority. Thus they had little need to exchange with others 'One he has a family. He has got car so if I need help then he can drive me. But he can't come too much. He has family, children'.

Sayid would not ask them for help because he felt he had nothing to exchange. Instead he continued to seek to cultivate deeper friendships with individuals he met at work in order to develop the intimacy he needed to share his feelings, despite calling them 'fast friends'. To some extent these individuals, all without family in the UK and connections back home engaged in norm-based reciprocity by spending time together and distracting each other from isolation and boredom.

\section{Over-reciprocation}

Some individuals actively sought opportunities to help others because offering help was an important aspect of their cultural or religious identity which gave them a sense of purpose. They engaged in either formal or informal volunteering often connected to their place of worship. Such actions were not necessarily a precursor to the development of social relationships and indeed there were often no expectations of exchange, merely of sacrifice but unlike informal reciprocity they exchanged with individuals who were known to them. But the feelings of self-worth experienced following their seemingly unreciprocated gift of time or care meant that they did in fact gain resources: namely self-esteem and a sense of being in control perhaps after a period where they had lost agency. There were many examples of this type of activity from the Pakistani refugee who devoted his spare time to helping the Ahmadi 
community 'It keeps me busy. Although there is some stress it is a way to feel relaxed. You organise things for the children who will be the future of the nation'. A Zimbabwean refugee who felt very isolated and said she had no friends whatsoever attended church each week and helped 'this other woman .... It is voluntary work. To help her with the shopping and to clean the house because she can't do that on her own. I met her at church’ but expected no reciprocity.

\section{No reciprocity}

We identified just two respondents who did not engage in any reciprocal exchanges both of whom were in the UK with their families. Olisa, originally from Nigeria, lived with his Japanese wife and their children and spent all of his time with them when he was not at work. He was clearly wary of building friendships and wanted to focus on conserving resources within his family unit. It is possible that he was conserving resources until he is better established but also that the closeness of his relationship with his wife meant he had no need to engage in reciprocity outside of his family unit. He gained the resources he needed in terms of intimacy, meaningfulness and a positive outlook and self-esteem through those close family relationships. He appeared wary about the risk-taking associated with sacrifice and felt exchange had little to offer

Well first of all. I am a very busy guy. With my job and my family. Before I don't like to make too much friends. Throughout my life. I don't want trouble. Sometimes friends are very helpful. Sometimes they are very dangerous. They can lead you into a life you don't want to be.

I don't like relying on people. It is like a stigma to me. I don't like it. I don't like it. Honestly. I like to be myself. That is my life. 
Raza originated from Pakistan but had lived all over Europe. He lived in the UK with his family and had a good job. He had an uncle living nearby who offered some assistance but otherwise appeared self-sufficient. He explained that he knew how to navigate UK institutions and had no need for help. Like Olisa he did not want to make friends because he wanted to focus his efforts upon finding his family a nice home and on settling in. Raza appeared to be conserving resources by avoiding the exchange that would be an inevitable part of network development. Later he planned to connect with people 'when I am settled, and then we can go to other cities. See family. But not now'.

Olisa and Raza shared some characteristics. They had decent jobs and housing, were either living with someone familiar within the UK or were familiar with institutions themselves, they had families with them and spoke English well. As such it could be argued that they had less need for resources than our other interviewees. They had homes, intimacy, knowledge, companionship, food and financial security - many of the integration resources outlined by Ager \& Strang (2008) and the resources Hobfoll identified as important. They reported no isolation, stress, uncertainty or loneliness and enjoyed the responsibility and associated self-esteem of taking care of their families. Their reluctance to engage in reciprocity may relate to the absence of need for further resources at least at this stage in their life or may simply relate to a lack of time given they were working and had small children.

\section{Discussion and Conclusions}

Focussing upon the experiences of new migrants moving to superdiverse cities we bring new insight into the ways in which new migrants engage in different forms of reciprocation to develop social connections and subsequently access resources. In particular we show how such reciprocity facilitates access to affective, psychological and spiritual resources that are frequently neglected in integration theory (see 
Grzymala-Kazlowska \& Phillimore 2017). Such resources are important in the mitigation of post-migration bereavement and acculturative stress which have been shown to undermine integration processes (Berry, 2005; Phillimore 2011). Considering how different forms of reciprocity can facilitate access to integration resources moves integration theory in a completely new direction focusing on agency in the form of exchange of resources rather than identity (Bhatia and Ram 2010) or functional indicators (Ager and Strang 2008). A reciprocity lens sheds new light on the relationship between social networks and integration while allowing us to identify an extended range of resources from those generally associated with integration (see also Ryan this issue).

The types of reciprocity engaged in varied over time, culture, gender and migration route. We find Godbout's notion of informal reciprocity particularly useful in understanding how resources were circulated between relatively established and newer migrants or other strangers. Places of worship, ESOL classes and libraries were important spaces in which migrants met for the first time and began to develop relationships based on exchange of the resources they gradually acquired in their new country. On the whole the longer individuals had been in the UK, the more resources they acquired but, as Bhatia and Ram (2009) argue, acquisition of resources was not a teleological process and some individuals did not report gains over time or experienced further losses.

Knowledge of local language, culture and institutions were key resources with considerable exchange value. Those who learned to navigate the system and had networks that yielded social capital in the form of functional resources such as housing and employment were in a position to initiate exchanges. More established migrants offered these resources to newest migrants as part of the open ended, non-specific, 
exchange as counterparts for the very same resources they received when they were newcomers. The act of returning these resources fulfilled their side of the unspoken bargain but it also enabled individuals to, as Hobfall (2011) suggests, replace resources lost through the process of migration and becoming a newcomer. Helping those less knowledgeable than themselves allowed reacquisition of agency, status, prestige and purpose. In this way offering informal reciprocity to strangers appeared to function as a marker of progress in their integration process and a mechanism to regain identity.

Those engaging in more norm-based acts of reciprocity, in particular female marriage migrants, appeared to share resources and offer each other social support which Ryan (2008) connects to the emergence of social capital. Through ongoing sharing they began to build intimacy, a resource lost in the act of migration when they were separated from friends and family in their country of origin. Such relationships provided companionship and loyalty but also facilitated access to integration indicators such as health services and employment (Ager \& Strang 2008) thereby aiding the replacement or substitution of other lost resources. Migration status, levels of residential stability and the availability of time impinged on willingness and ability to exchange. Forced migrants were in the weakest positions because they arrived with few resources and were more likely to under-reciprocate than people of other migration statuses. Dispersed to unfamiliar areas and not permitted to study or work their ability to gain institutional knowledge and build networks based on reciprocity was limited. While Nahun-Shani \& Bamberger (2011) show this can be stressful, and even cause further resource loss by undermining self-esteem, some individuals rationed their access to those with resources for fear of violating exchange norms. The importance of normbased reciprocity as described by Simmel and Mauss may be culturally specific as we see in the case of our Somali respondent who embraced the resources he was offered as 
part of the Somali culture of sharing. The open-ended arrangements described by Bernard et al. (2007) were socially acceptable for him and did not appear to undermine his sense of agency.

On the whole we find that reciprocal relationships are crucial but are complex, manifold and non-linear. The forms of reciprocity that new migrants enter into are shaped by their previous migration experience, legal status, presence of family, employment and language ability as well as the nature of the local context into which they move. Rather than conserving resources as predicted by Hobfoll (2011) it appeared those with the least resources had the greatest need for exchange (or perhaps the least choice in whether to engage in exchange). Only through exchange that was based around spending time or offering care or knowledge could they regain or substitute important lost resources: intimate relationships, companionship, self-esteem and purpose. As Bernard et al. (2007) suggests the act of reciprocation appeared in some cases to be more important than the nature of the resources exchanged. Being able to offer resources to a stranger who will never repay them signified the possession of agency the importance of which may outweigh the desire for defensiveness. Reciprocity in all forms except under-reciprocity highlights that, after a period without resources, the newcomer now has resources to sacrifice: the act of giving is a practice of expressing regained agency. Perhaps the more established migrants: those with a partner, a family, a job and a home have less need to take the risk of exchanging because they can already access the integration resources they need and have less time available for exchange. Certainly in our study they were the most defensive, contradicting Hobfoll’s ideas.

While our study was based upon the experiences of new migrants solely resident in England, reciprocity emerged as important for a very varied set of respondents. We 
believe this suggests that reciprocity as a facilitator of integration is likely to be important in other superdiverse geographical contexts and in different migration regimes. Having identified, for the first time, the significance of reciprocity in integration we argue further empirical and conceptual work is needed to examine the relationship between resource exchange and integration processes, the circumstances in which different kinds of exchange are possible, and the outcomes of those exchanges. This would include consideration of which kinds of resources aid integration and whether some have the potential to be anti-integrative as well as the interconnections between social networks and capital, functional and psychological resources. It is important to acknowledge the distinction between reciprocity as a material practice and reciprocity as topos in the narrative construction of migration experiences - the extent to which individuals construct themselves as reciprocators may differ from their actual behaviours. Migrants' constructions of reciprocity could be an important focus for future integration studies. Finally it is necessary to consider the ways in which integration and social cohesion policies and practices facilitate or undermine reciprocity and associated access to resources which may vary across contexts.

\section{References}

Abdulahad, R., Graham, J.R., Montelpare, W.J., Brownlee, K., 2012. Social Capital: Understanding Acculturative Stress in the Canadian Iraqi-Christian Community. Br. J. Soc. Work bcs160. doi:10.1093/bjsw/bcs160

Ager, A., Strang, A., 2008. Understanding Integration: A Conceptual Framework. J. Refug. Stud. 21, 166-191. doi:10.1093/jrs/fen016

Anthias, P.F., Cederberg, D.M., 2009. Using Ethnic Bonds in Self-Employment and the Issue of Social Capital. J. Ethn. Migr. Stud. 35, 901-917.

doi:10.1080/13691830902957692 
Barwick, C., 2015. Are Immigrants Really Lacking Social Networking Skills? The Crucial Role of Reciprocity in Building Ethnically Diverse Networks. Sociology 0038038515596896. doi:10.1177/0038038515596896

Benight, C.C., Ironson, G., Klebe, K., Carver, C.S., Wynings, C., Burnett, K., Greenwood, D., Baum, A., Schneiderman, N., 1999. Conservation of resources and coping self-efficacy predicting distress following a natural disaster: A causal model analysis where the environment meets the mind. Anxiety Stress Coping 12, 107-126. doi:10.1080/10615809908248325

Bernard, P., Charafeddine, R., Frohlich, K.L., Daniel, M., Kestens, Y., Potvin, L., 2007. Health inequalities and place: A theoretical conception of neighbourhood. Soc. Sci. Med., Placing Health in Context 65, 1839-1852. doi:10.1016/j.socscimed.2007.05.037 Berry, J.W., 2009. A critique of critical acculturation. Int. J. Intercult. Relat. 33, 361371.

Berry, J.W., 2005. Acculturation: Living successfully in two cultures. Int. J. Intercult. Relat., Special Issue: Conflict, negotiation, and mediation across cultures: highlights from the fourth biennial conference of the International Academy for Intercultural Research 29, 697-712. doi:10.1016/j.ijintrel.2005.07.013

Bhatia, S., Ram, A., 2009. Theorizing identity in transnational and diaspora cultures: A critical approach to acculturation. Int. J. Intercult. Relat., Critical Acculturation Psychology 33, 140-149. doi:10.1016/j.ijintrel.2008.12.009 Bilecen, B., 2014. International student mobility and transnational friendships. Palgrave Macmillan.

Bloemraad, I., Korteweg, A., Yurdakul, G., 2008. Citizenship and Immigration: Multiculturalism, Assimilation, and Challenges to the Nation-State. Annu. Rev. Sociol. 34, 153-179. doi:10.1146/annurev.soc.34.040507.134608 